children with cystic fibrosis. Am J Clin Nutr 1988;48(6): I362-1366.

10. Oakie S. Fed up! Winning the war against childhood obesity. Washinton DC: Joseph Henry press, 2005.

II. Hernández B, García-Feregrino R, Rivera JA, Sepúlveda-Amor J, Ramírez-Silva CL, Monterrubio EA et al. Factores asociados con sobrepeso y obesidad en niños mexicanos de edad escolar: resultados de la Encuesta Nacional de Nutrición de 1999. Salud Publica Mex 2003;45(supl 4):S55I-S557.

12. Cosío JA, Lázaro JA, Pizano U. Prevalencia del sobrepeso y obesidad determinada por medio del índice de masa corporal en niños y niñas de la escuela primaria "Niños Héroes" de Villa de Álvarez, Colima (tesis). Colima: Universidad de Colima, 2006

13. Nicklas TA, Dwyer J, Feldman HA, Luepker RV, Kelder SH, Nader PR. Serum cholesterol levels in children are associated with dietary fat and fatty acid intake. Journal of American Diet Association 2002; I02(4):5I I-5 I7.

14. Sahota P, Rudolf MCJ, Dixey R, Hill AJ, Barth $\mathrm{JH}$, Cade J. Randomised controlled trial of primary school based intervention to reduce risk factors for obesity. British Medical Journal 200I;323:I-5

15. Kelley GA, Kelley KS. Aerobic exercise and lipids and lipoproteins in children and adolescents: A meta-analysis of randomized controlled trials". Atherosclerosis 2007;191(2):447-453.

16. Caballero B, Clay T, Davis SM, Ethelbah B, Rock BH, Lohman T et al. Pathways: a schoolbased, randomized controlled trial for the prevention of obesity in American Indian schoolchildren. American Journal of Clinical Nutrition 2003;78(5): 1030-1038.

\section{Etiología de la sepsis neonatal en una unidad hospitalaria de segundo nivel}

Señor editor: el recién nacido que requiere manejo en unidades de cuidados intensivos tiene mayor riesgo de presentar sepsis, con una mayor incidencia en países menos industrializados. No obstante, la mayor información proviene de países industrializados en donde, por ejemplo, Estados Unidos reporta una incidencia de 1 a $8 / 1000$ recién nacidos vivos, ${ }^{1}$ en contraste con lo reportado en países en vías de desarrollo como India, Pakistán y Tailandia, donde la incidencia es de 2.4 a 16/1 000 nacidos vivos.

En nuestro medio si bien la información es limitada, en el Instituto
Nacional de Perinatología (INP) se reporta una incidencia de 19/1 000 recién nacidos vivos. ${ }^{2}$ Los microorganismos involucrados muestran variaciones significativas en relación con el tiempo de presentación de la infección (temprana o tardía), ${ }^{3}$ así como si existe antecedente de prematurez, uso de ventilador, uso de catéteres intravasculares y, en general, procedimientos de tipo invasivo para el diagnóstico y tratamiento de los recién nacidos, aunado a las variaciones regionales, inclusive interinstitucionales. ${ }^{4}$ El S. beta hemolítico del grupo B constituye en Estados Unidos y Europa el agente etiológico más frecuente, mientras que en países menos industrializados los microorganismos gram negativos siguen siendo los predominantes. En el Instituto Nacional de Pediatría la K. pneumoniae ${ }^{5}$ constituye el microorganismo más frecuente, similar a lo reportado en otras instituciones. ${ }^{6}$

El objetivo de este estudio fue reportar los agentes etiológicos, edad de presentación, complicaciones y mortalidad en niños con diagnóstico de egreso de sepsis neonatal hospitalizados en el Hospital General de Zona 1A Los Venados del Instituto Mexicano del Seguro Social (IMSS) entre el 1 de enero de 2004 y el 30 de septiembre de 2005.

Se revisaron los expedientes clínicos de recién nacidos con diagnóstico de egreso de sepsis neonatal que ingresaron a la unidad de cuidados intensivos, cunero patológico y de prematuros del hospital.

Se consideró probable sepsis neonatal en aquellos niños de 30 días de edad o menores que presentaban tres o más de los siguientes datos clínicos: irritabilidad, hipotermia, fiebre, vómito, succión débil, distensión abdominal, presencia de residuo gástrico, ictericia, apneas, visceromegalia, aunado a una o más alteraciones en la biometría hemática sugestivas de infección bacteriana, como leucocitosis mayor a 20000 leucopenia (leucocitos $\leq 4000$ células $/ \mathrm{mm}^{3}$ ) relación banda / neutrófilos $>0.20$, plaquetas $<100.000$, velocidad de sedimentación globular (VSG) $\geq 20 \mathrm{~mm} / \mathrm{hr}$, proteína C reactiva $>16 \mathrm{mg} \%$, presencia de granula- ciones tóxicas o neutrófilos vacuolados en el frotis de sangre periférica. Se consideró sepsis documentada cuando el paciente reunía los criterios antes mencionados y contaba, además, con la identificación microbiológica en el hemocultivo. Se mantuvo la confidencialidad de la información.

Se analizó la información en un paquete en SPSS versión 11.0. La información obtenida se describió mediante promedio \pm desviación estándar en el caso de variables numéricas continuas con distribución gaussiana, para lo cual se calculó sesgo, kurtosis y prueba de Kolmogorov Smirnov. Las variables numéricas sesgadas se describieron con mediana con mínimos y máximos y las variables categóricas con frecuencia relativa (porcentajes). Para la comparación de variables numéricas se utilizó la prueba $t$ de Student o U de Mann-Whitney para aquellas variables con varianzas diferentes o a través de ANOVA de una vía o Kruskal Wallis en el caso de comparación de más de dos medias, y la prueba exacta de Fisher, para la contrastación de variables categóricas; se consideró significancia estadística una $p=0<0.05$.

Se compararon las variables categóricas a través de ji cuadrada o prueba exacta de Fisher. Las variables numéricas continuas se compararon a través de la prueba $t$ de Student o U de Mann-Whitney. Se consideraron diferencias estadísticamente significativas las asociadas a un valor de $p \leq 0.05$.

Encontramos que durante este periodo nacieron 3633 niños, de los cuales $305(8 \%)$ fueron ingresados a la unidad de cuidados intensivos neonatales (UCIN), $2100(58 \%)$ a cuneros patológicos y 1228 (34\%) a binomio madre-hijo. De los 2405 recién nacidos que permanecieron en el hospital, $200(8.3 \%)$ tuvieron diagnóstico de egreso de sepsis neonatal, de los cuales sólo 124 cumplían con los criterios establecidos para el diagnóstico, por lo que fueron incluidos en el análisis.

La distribución de acuerdo con edad gestacional fue similar en ambos grupos: 66 niños fueron prematuros (53\%), 55 correspondieron a niños de término $(44 \%)$ y tres fueron de postérmino $(2 \%)$; 
69 correspondieron al género masculino (64\%). El 99\% tenía peso de acuerdo con la edad gestacional.

No se observaron diferencias en relación con el tipo de parto (cesárea o parto eutósico) y edad gestacional. Veintitrés pacientes tuvieron antecedente de hipoxia perinatal $(19 \%)$; se presentaron sólo seis pacientes con puntuación Apgar menor a $5(4 \%)$.

El promedio de edad al inicio de la sintomatología fue de cinco días. Ciento dos casos $(82 \%)$ correspondieron a sepsis temprana. Las manifestaciones clínicas más frecuentes fueron dificultad respiratoria con $98(79 \%)$, ictericia $55(44 \%)$, desequilibrio ácido base $21(17 \%)$, enterocolitis necrotizante $18(16 \%)$, residuo gástrico $17(14 \%)$, rechazo al alimento 15 (12\%), distensión abdominal $32(26 \%)$. Los principales órganos afectados en los 124 recién nacidos fueron: pulmón 60 (45\%), metabólico $28(21 \%)$ y digestivo $27(20 \%)$.

La mayoría de los pacientes (91) presentó leucocitosis con una mediana de 22500 (límites de $2100 / \mathrm{mm}^{3}$ a $\left.60000 / \mathrm{mm}^{3}\right), 23(19 \%$ ) de leucopenia y el valor de la hemoglobina en $95(77 \%)$. Se encontraron cifras normales entre $14 \mathrm{y}$ 17 gr. La cifra de plaquetas en los 124 recién nacidos fue de 5500 hasta 501000 con una mediana de 160 000. La velocidad de sedimentación globular (VSG) se reportó sólo en 20 pacientes con una media de $17 \mathrm{~mm} / \mathrm{h}$. La relación banda neutrófilo fue de 0.30 . La proteína $C$ reactiva en todos casos fue negativa.

El hemocultivo fue positivo en 36 pacientes $(29 \%)$ con discreto predominio de los gram positivos en 19 casos (52\%).

El esquema antimicrobiano utilizado con mayor frecuencia fue la combinación de un betalactámico y una cefalosporina de tercera generación (ceftriaxona más amikacina) en 83 pacientes $(67 \%)$, seguida de un glucopéptido (vancomicina) en combinación con aminoglúcosido en 15 casos $(12 \%)$, cefalosporina de tercera o cuarta generación en 22 pacientes y con menos frecuencia otros. La duración promedio del tratamiento fue de ocho días.
La estancia hospitalaria promedio fue de 18 días. Veintidós pacientes fallecieron $(17 \%)$.

$\mathrm{Al}$ analizar no hubo diferencia estadísticamente significativa al comparar los niños de término con los pretérmino con sepsis. No hubo diferencia significativa entre los que murieron con sepsis temprana y los que murieron son sepsis tardía. Se realizó prueba de Fischer entre los niños de término y de pretérmino con sepsis temprana y tardía con $p<0.05$.

Se observó que la incidencia de sepsis neonatal en esta población estudiada fue de 3.4/ 1000 recién nacidos vivos, menor a la incidencia de 19/1000 notificada en el $\mathrm{INP}^{2}$ lo cual pudiera estar relacionado con que en el Instituto habitualmente se atienden pacientes de alto riesgo. ${ }^{7}$ Se encontró predominio en el sexo masculino en $64 \%$ de los casos. La teoría propuesta sugiere un factor de susceptibilidad relacionado con un gen localizado en el cromosoma $X$ involucrado con la función del timo y síntesis de inmunoglobulinas por lo tanto la niña al poseer dos cromosomas $X$ tiene mayor resistencia a la infección. ${ }^{8}$

Otro factor de riesgo ya descrito es el recién nacido prematuro. En este estudio $64 \%$ pertenecieron a dicha categoría. Esos niños están más expuestos a padecer sepsis por inmadurez fisiológica del sistema humoral y celular con disminución del complemento sérico, en la quimiotaxis, fagocitosis, opsonización, adherencia disminuida de neutrófilos y falta de anticuerpo de tipo específico, lo que predispone a un sinnúmero de infecciones, ${ }^{9}$ así como la estancia prolongada hospitalaria con la subsiguiente prolongación de exposición.

Se ha descrito que la presentación de sepsis temprana es de dos tercios y en este estudio también lo fue con 102 casos (82\%). Los niños con sepsis temprana tuvieron como factor de riesgo prematurez en $66(64 \%)$, parto distócico $6(6 \%)$ hipoxia moderada a severa $23(19 \%)$. Los datos clínicos encontrados fueron dificultad respiratoria, ictericia, distensión abdominal, rechazo al alimento, residuo gástrico, lo que concuerda con lo reportado internacionalmente; $81 \%$ de los pacientes cursaron con leucocitosis. ${ }^{10}$ La proteína C reactiva fue negativa en todos los casos, la cual, como reactante de fase aguda, era de esperar que se encontrara elevada en las primeras horas de infección, ${ }^{11}$ aunque diversos autores concluyen que no existe un examen de laboratorio con suficiente sensibilidad y especificidad diagnóstica de sepsis neonatal; por lo tanto, lo que debe evaluarse para una adecuada prescripción es el conjunto de datos clínicos y paraclínicos. ${ }^{12}$ Los sitios de infección primaria fueron pulmonar y gastrointestinal. En este estudio fue probablemente por el uso de ventilación mecánica, situación que ha sido descrita por diversos autores. Se encontró aislamiento bacteriológico en 29 por ciento. El INP reporta 19\%, Jashi informa 25\%, Moreno 28\% y Escobar 35 por ciento. 6

Al comparar a los niños que fallecieron prematuros y de término con sepsis temprana y con sepsis tardía no hubo diferencia significativa, por lo que la conclusión en este estudio es que los niños están expuestos a los mismos riesgos y tienen la misma posibilidad de adquirir sepsis e inclusive fallecer por dicha enfermedad.

Dra. María de Lourdes Patricia Ramírez Sandoval Pediatría, Hospital General de Zona IA, Los Venados, IMSS

Municipio Libre 28, Col. Portales, Delegación Benito Juárez, México, DF Pediatría, Hospital Regional Adolfo López Mateos, ISSSTE

Correo electrónico: lourpaty@yahoo.com.mx

Dra. Mercedes Macías Parra Instituto Nacional de Pediatra

Dr. Federico Lazcano Ramírez Servicios de Salud del Distrito Federal.

\section{Referencias}

I. Jafari HS, McCracken GH. Sepsis and septic shock: a review for clinicians. Pediatr Infect Dis J 1992; | 1:739-749.

2. Normas y Procedimientos de Neonatología. México: Instituto Nacional de Perinatología, 1998:222-224

3. Lares Al, Reyes BM, Ramírez SP.

Farmacocinética de los antimicrobianos En: 
González SN, Saltigeral P, Macias PM. Infectologia Neonatal. México:Trillas, 1997:347-350.

4. Rubén A Villalaz B. Sepsis en el recién nacido En: Xavier Sáez Llorens. Sepsis Neonatal. México: McGraw-Hill, 2002:31-37.

5. Rodríguez-Weber MA. López-Candiani C, Arredondo-García JL, Gutiérrez-Castrellón P, Sánchez-Arriaga F. Morbilidad y mortalidad por sepsis neonatal en un hospital de tercer nivel de atención. Salud Publica Mex 2003;45:90-95.

6. Ramírez S, Hernández B, Carlos O. Brote Nosocomial por Klebsiella ozaenae en cuneros patológicos del hospital general de zona no. 32 IMSS. Rev Enf Infec Pediatr 2002, 16(6I):4-10.
7. St Geme JW, Murray DL, Carter J, Hobel C], Leake RD,Anthony BE Perinatal bacterial infection after prolonged rupture of amniotic membranes: an analysis of risk and management. ] Pediatr 1984;104(4):608-613.

8. Rosalinda-Tristan E. En: Xavier Sáez-Llorens. Sepsis Neonatal. México: McGraw-Hill, 2002:I I-17. 9. Nieto-Guevara J, Sáez-Llorens X. Sepsis y choque séptico En: González SN, Saltigeral SP, Macías PM. Infectología Neonatal. 2a ed. México: McGraw-Hill, 2006: 14-26.
I0. Escobar G], Li D,Armstrong MA, Gardner $\mathrm{MN}$, Folck BF, et al. Neonatal sepsis workups in infants $>2000$ grams at birth:A population-based study. Pediatrics 2000; 106(2):256-263.

II. Hajiehe Borna, Sedigheh Borna:Value of laboratory tests and $C$ reactive protein in the detection of neonatal sepsis. The Internet Journal of Pediatrics and Neonatology. 2005;5(2).

12. Anwer S, Mustafa S. Rapid identification of neonatal sepsis.J Pak Med Assoc 2000;50(3):94-98. 